\title{
Implicit Method and Stability of Dynamic Grid for Nonlinear Schroedinger equation
}

\author{
Agah D. Garnadi
}

\begin{abstract}
This work studies consists of two parts. The first part studying implicit method for Nonlinear Scrhoedinger equation, while the second part study the Backward Euler in dynamic Grid methods for the Nonlinear Schroedinger with critical power nonlinearity. A first-order implicit backward Euler method is studied for applicaton to nonlinear Schroedinger with critical power depicting blow-up. The focal point of the paper is to examine the efficiency and reliability of the method in handling of possibility of blow-up to capture the asymptotic behavior. Using field of values, we observe the distortion of eigen-values inclusion of spatial grid discretization.
\end{abstract}

MSC 2010: 65M06, 65M20; 65Y05, 65Y20

Keyworsds. Numerical methods, Implicit, Backward Euler, Nonlinear Schroedinger, critical power, finite time solution, dynamic grid. 


\section{On Implicit Method for Non-Linear Schrödinger Equation \\ II Stability of Backward Euler in Dynamic Grid Methods for Non-Linear Schrödinger Equation \\ Notes 1}

The main objective of this note is to investigate the eigenvalues region inclusion of spatial discretization of Linear Schrödinger Equations over uniform spacing. We extends the results of this note to non-uniform grid spacings in the future notes.

Let us consider 1-D linear Schrödinger with advection as our model equation :

$$
u_{t}=i u_{x x}+c(x) u_{x}
$$

where $u$ is periodic with periods $2 \pi$, and $c(x)$ is a real-valued function, given $2 \pi$ periodic initial values:

$$
u(x, 0)=f(x), f(x)=f(x+2 \pi)
$$

To locate the eigenvalues of discrete operator, we use a refinement of Gershgorincircle like inclusion method. We cites only some definition and properties that considered important to our needs. For further details see Horn \& Johnson [HJ91].

1. Eigenvalues Inclusion Region. Let $A \in M_{n}(C), n \times n$ matrix with complex entries.

\section{Definition}

Field of values of $\mathrm{A}$ is the region in complex plane defined as:

$$
F(A)=\left\{x^{*} A x:\|x\|=1\right\}
$$

Let $\sigma(A)$ denotes the set of spectra of A.

Properties:

i. $F(A+B) \subset F(A)+F(B)$

ii. $\sigma(A) \subset F(A)$

The addition is taken as Minkowski set addition, that is point-wise addition of corresponding sets.

\section{Definition}

Deleted row-sum :

$$
R_{k}^{\prime}(A)=\sum_{j=1, j \neq k}\left|a_{k, j}\right|, k=1, \cdots, s
$$

Deleted column-sum:

$$
C_{j}^{\prime}(A)=\sum_{k=1, j \neq k}\left|a_{k, j}\right|, j=1, \cdots, s
$$

Averaged Gershgorin-radii

$$
g_{j}(A)=\frac{1}{2}\left[R_{j}^{\prime}(A)+C_{j}^{\prime}(A)\right], j=1, \cdots, s
$$

generalized Gershgorin region

$$
G_{F}(A)=\operatorname{co}\left[\cup_{j=1}^{n}\left\{z:\left|z-a_{j j}\right| \leq g_{j}(A)\right\}\right]
$$


which is a convex hull of union of Gershgorin-circle like with averaged deleted row-sum and deleted column-sum.

The relation between the field-of-values and generalized Gershgorin region given by the following properties.

Properties:

i. $F(A) \subset G_{F}(A)$

2. Discretization on Uniform Grid. We want to solve the above equation using finite difference approximation. For this purposes, let us divides the interval $[0,2 \pi]$ into $2 \mathrm{~N}$-sub interval with uniform gridsize $h=\frac{2 \pi}{2 N+1}, \mathrm{~N}$ a natural number, this subdivision introduces gridpoints $x_{\nu}=\nu h, \nu=0,1, \cdots, 2 N$, and grid functions $u_{\nu}(t)=u\left(x_{\nu}, t\right)$. Using central differences for second and first partial derivatives we obtain:

$$
\begin{array}{r}
\frac{d u_{\nu}}{d t}=i \frac{u_{\nu+1}-2 u_{\nu}+u_{\nu-1}}{h^{2}}+c_{\nu} \frac{u_{\nu+1}-u_{\nu-1}}{2 h} \\
u_{\nu}(0)=f\left(x_{\nu}\right)
\end{array}
$$

where $c_{\nu}=c\left(x_{\nu}\right)$ with periodicity conditions:

$$
u_{0}=u_{2 N+1}, u_{-1}=u_{2 N}
$$

In matrix form the discrete systems becomes:

$$
\begin{aligned}
\frac{d u}{d t}=i L_{h} u+C D_{0} u & =A u \\
u(0) & =f
\end{aligned}
$$

where :

and

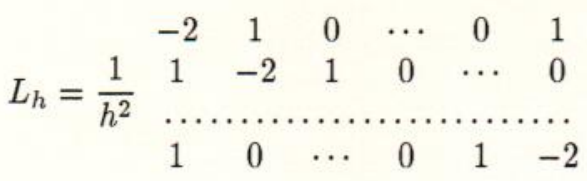

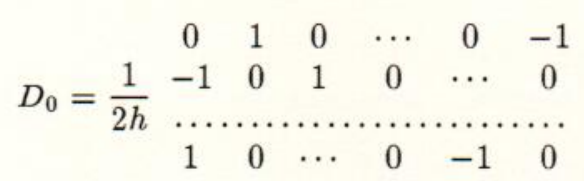

where $C$ is a diagonal matrix with entries : $C_{j j}=c\left(x_{j}\right)$. Observe that $L_{h}$ is a symmetric matrix while $D_{0}$ is a skew-symmetric matrix. Let us first consider the case $c(x)$ is a constant. It is easily be shown that the spatial discrete operator $A=\left\{i L_{h}+\right.$ $\left.c D_{0}\right\}$ is a skew-hermitean, so a normal matrix. Using discrete Fourier expansion, we obtain eigenvalues-eigenvectors pairs of this discrete operator :

$$
\begin{array}{r}
\left\{i L_{h}+c D_{0}\right\} \mathrm{e}^{i \omega x}=\frac{i}{h}\left(-\frac{4}{h} \sin ^{2}\left(\frac{\omega h}{2}\right)+c \sin (\omega h)\right) \mathrm{e}^{i \omega x}, \\
\omega=-N, \ldots,-1,0,1, \ldots, N
\end{array}
$$

Unfortunately, if $c(x)$ is not constant, the discrete operator in general is not a normal matrix anymore. 


\section{Eigenvalues Region}

Now, we calculates eigenvalues region inclusion, for non-constant $c(x)$, which in this case results a diagonal with entries $C_{j j}=c\left(x_{j}\right)$. So we consider $A=\left\{i L_{h}+C D_{0}\right\}$. Using the properties that :

$$
\sigma(A) \subset F(A) \subset\left\{F\left(i L_{h}\right)+F\left(C D_{0}\right)\right\}
$$

It is easily obtained that

$$
F\left(i L_{h}\right)=i F\left(L_{h}\right)=-i\left[0, \lambda_{M}\right]
$$

where $\lambda_{M}$ is the largest modulus of eigenvalue of $L_{h}$. While to estimate the field of values $F\left(C D_{0}\right)$ we use its inclusion by the generalized Gershgorin region:

$$
F\left(C D_{0}\right) \subset G_{F}\left(C D_{0}\right)
$$

By direct calculation, we obtain deleted row-sum

$$
\begin{array}{r}
R_{k}^{\prime}\left(C D_{0}\right)=\sum_{j=1, j \neq k}\left|c_{k k} d_{k, j}\right|, k=1, \cdots, s \\
=\frac{2\left|c_{k k}\right|}{2 h}, k=1, \cdots, s
\end{array}
$$

and deleted column-sum

$$
\begin{array}{r}
C_{j}^{\prime}\left(C D_{0}\right)=R_{j}^{\prime}\left(-D_{0} C\right) \\
=\sum_{k=1, j \neq k}\left|d_{j, k} c_{k k}\right|, j=1, \cdots, s \\
=\frac{\left|c_{j-1, j-1}\right|+\left|c_{j+1, j+1}\right|}{2 h}, j=1, \cdots, s
\end{array}
$$

so we get averaged Gershgorin radii

$$
\begin{gathered}
g_{j}\left(C D_{0}\right)=\frac{1}{2}\left[R_{j}^{\prime}\left(C D_{0}\right)+C_{j}^{\prime}\left(C D_{0}\right)\right], j=1, \cdots, s \\
=\frac{1}{2} \frac{2\left|c_{k k}\right|}{2 h}+\frac{\left|c_{j-1, j-1}\right|+\left|c_{j+1, j+1}\right|}{2 h}, j=1, \cdots, s
\end{gathered}
$$

which results for generalized Gershgorin region

$$
\begin{array}{r}
G_{F}\left(C D_{0}\right)=\operatorname{co}\left[\cup_{j=1}^{n}\left\{z:|z| \leq g_{j}\left(C D_{0}\right)\right\}\right] \\
=\operatorname{co}\left\{z:|z| \leq \max \left\{\max \left\{\frac{\left|c_{j j}\right|}{h}\right\}, \max \left\{\frac{\left|c_{j-1, j-1}\right|+\left|c_{j+1, j+1}\right|}{2 h}\right\}\right\}\right\} \\
=\left\{z:|z| \leq \max \left\{\frac{\left|c_{j j}\right|}{h}\right\}\right\}
\end{array}
$$

by using the fact that diagonal entries of $C D_{0}$ are zero. Combining this result with field of values of $i L_{h}$, we obtain

$$
\sigma(A) \subset F(A) \subset-i\left[0, \lambda_{M}\right]+\left\{z:|z| \leq \frac{\left|c_{j j}\right|}{h}\right\}
$$

this is an oval region with axis ratio $O\left(\frac{1}{h}\right)$ and its major axis lies on imaginary axis. 
From the above results, we obtains bounds of the spectral radius of this matrix in the term of eigenvalues of operator $L_{h}$ and $D_{0}$ :

$$
\rho\left(i L_{h}+C D_{0}\right) \leq \lambda_{M}+c_{M} \mu_{M}
$$

where $\lambda_{M}$ and $\mu_{M}$ are maximum modulus of eigenvalues of $L_{h}$ and $D_{0}$ respectively, and $c_{M}$ is the maximum modulus of $\mathrm{C}$ entries.

I

\section{Comments on Discretization over non-uniform Grid}

The objective of this note is to investigate the eigenvalues region inclusion of spatial discretization of Linear Schrödinger Equations over non-uniform spacing. We extends the results on uniform grids given in the previous notes to non-uniform grid spacings. We follow notation given previously.

Let us consider 1-D linear Schrödinger as our model equation :

$$
u_{t}=i u_{x x}
$$

where $u$ is periodic with periods $2 \pi$, given $2 \pi$-periodic initial values:

$$
u(x, 0)=f(x), f(x)=f(x+2 \pi)
$$

\section{Preliminary}

To extends some results on uniform grid to non-uniform grid, we requires following lemma. For proofs see Axelsson [Ax92].

\section{Lemma} Then:

Let A, B symmetric matrices, $\lambda_{i}(A)$ denotes $i$-th eigenvalues in increasing order.

(a). If $\lambda_{\max }(B)$ is non-negative and $\mathrm{A}$ is positive definite,then

$$
\lambda_{i}(A B) \leq \lambda_{i}(A) \lambda_{\max }(B)
$$

(b). If $\lambda_{\min }(B)$ is non-negative and $\mathrm{A}$ is positive definite,then

$$
\lambda_{i}(A) \lambda_{\min }(B) \leq \lambda_{i}(A B)
$$

\section{Discretization on Non-Uniform Grid}

Let $\left\{x_{j}\right\}$ denotes a non-uniform grid obtained from a uniform computational grid $\left\{\xi_{j}\right\}$ with spacing grid $h$, using preserving index transformation. Corresponds to nonuniform grid $\left\{x_{j}\right\}$ denotes $h_{m}=\min \left\{h_{j} ; h_{j}=\left(x_{j+1}-x_{j}\right)\right\}$ and $h_{M}=\max \left\{h_{j} ; h_{j}=\right.$ $\left.\left(x_{j+1}-x_{j}\right)\right\}$. Approximating the second and first partial derivatives using center differencing [Fl88], i.e :

$$
\begin{gathered}
\frac{\partial^{2} u}{\partial x^{2}}=\left\{\frac{2 h}{x_{j+1}-x_{j-1}}\right\}^{2} \times \\
\left\{\frac{u_{j+1}-2 u_{j}+u_{j-1}}{2 h^{2}}-\frac{h_{j}-h_{j-1}}{h^{2}} \frac{u_{j+1}-u_{j-1}}{h_{j}+h_{j-1}}\right\} \\
=\left(D_{\xi}^{-1}\right)^{2} \times\left\{L_{h}-D_{\xi \xi} D_{\xi}^{-1} D_{0}\right\} u
\end{gathered}
$$


where $D_{\xi \xi}$ and $D_{\xi}$ are diagonal matrices with entries : $\left(\frac{x_{j+1}-2 x_{j}+x_{j-1}}{h^{2}}\right)=\frac{h_{j}-h_{j-1}}{h^{2}}$ and $\left(\frac{x_{j+1}-x_{j-1}}{2 h}\right)=\frac{h_{j}+h_{j-1}}{2 h}$ respectively. The discrete problem, now can be written in the matrix form :

$$
\frac{d u}{d t}=i\left(D_{\xi}^{-1}\right)^{2}\left\{L_{h}-D_{\xi \xi} D_{\xi}^{-1} D_{0}\right\} u
$$

or after regrouping :

$$
\frac{d u}{d t}=i\left(D_{\xi}^{-1}\right)^{2} L_{h} u-i\left\{\left(D_{\xi}^{-1}\right)^{3} D_{\xi \xi}\right\} D_{0} u
$$

\section{Eigenvalues Region}

Now, we calculates eigenvalues region inclusion, consider

$$
A=i\left(D_{\xi}^{-1}\right)^{2} L_{h} u-i\left\{\left(D_{\xi}^{-1}\right)^{3} D_{\xi \xi}\right\} D_{0} u
$$

Following the method in previous notes, we use the properties that :

$$
\sigma(A) \subset F(A) \subset\left\{F\left(i \tilde{L}_{h}\right)+F\left(\tilde{C} D_{0}\right)\right\}
$$

where: $\tilde{L}_{h}=\left(D_{\xi}^{-1}\right)^{2} L_{h}$ and $\tilde{C}=-i\left\{\left(D_{\xi}^{-1}\right)^{2} D_{\xi \xi}\right\}$ with entries for $\tilde{C}$ :

$$
\begin{gathered}
\tilde{c}_{j j}=-i\left\{\left(\frac{2 h}{x_{j+1}-x_{j-1}}\right)^{3} \frac{\left(x_{j+1}-2 x_{j}+x_{j-1}\right)}{h^{2}}\right\} \\
=-i\left\{\left(\frac{2 h}{h_{j}+h_{j-1}}\right)^{3} \frac{\left(h_{j}-h_{j-1}\right)}{h^{2}}\right\}
\end{gathered}
$$

Using the lemma above, since $\left(D_{\xi}^{-1}\right)^{2}$ is symmetric positive definite and $-L_{h}$ is symmetric semi-positive definite, we obtain :

$$
F\left(i \tilde{L}_{h}\right)=i F\left(\tilde{L}_{h}\right)=-i\left[0, \tilde{\lambda}_{M}\right]
$$

where $\tilde{\lambda}_{M}=\max \left\{\left(\frac{2 h}{x_{j+1}-x_{j-1}}\right)^{2}\right\} \lambda_{M}$ with $\lambda_{M}$ is the largest modulus of eigenvalue of $L_{h}$. Now we estimate the field of values $F\left(\tilde{C} D_{0}\right)$ using its inclusion by the generalized Gershgorin region:

$$
F\left(\tilde{C} D_{0}\right) \subset G_{F}\left(\tilde{C} D_{0}\right)
$$

Directly calculating the deleted row-sum, we obtain:

$$
\begin{array}{r}
R_{k}^{\prime}\left(\tilde{C} D_{0}\right)=\sum_{j=1, j \neq k}\left|\tilde{c}_{k k} d_{k, j}\right|, k=1, \cdots, s \\
=\frac{2\left|\tilde{c}_{k k}\right|}{2 h}, k=1, \cdots, s
\end{array}
$$

and deleted column-sum

$$
\begin{array}{r}
C_{j}^{\prime}\left(\tilde{C} D_{0}\right)=R_{j}^{\prime}\left(-D_{0} \tilde{C}\right) \\
=\sum_{k=1, j \neq k}\left|d_{j, k} \tilde{c}_{k k}\right|, j=1, \cdots, s \\
=\frac{\left|\tilde{c}_{j-1, j-1}\right|+\left|\tilde{c}_{j+1, j+1}\right|}{2 h}, j=1, \cdots, s \\
5
\end{array}
$$


so we get averaged Gershgorin radii

$$
\begin{array}{r}
g_{j}\left(\tilde{C} D_{0}\right)=\frac{1}{2}\left[R_{j}^{\prime}\left(\tilde{C} D_{0}\right)+C_{j}^{\prime}\left(\tilde{C} D_{0}\right)\right], j=1, \cdots, s \\
=\frac{1}{2} \frac{2\left|\tilde{c}_{k k}\right|}{2 h}+\frac{\left|\tilde{c}_{j-1, j-1}\right|+\left|\tilde{c}_{j+1, j+1}\right|}{2 h}, j=1, \cdots, s
\end{array}
$$

which results for generalized Gershgorin region

$$
\begin{array}{r}
G_{F}\left(\tilde{C} D_{0}\right)=\operatorname{co}\left[\cup_{j=1}^{n}\left\{z:|z| \leq g_{j}\left(\tilde{C} D_{0}\right)\right\}\right] \\
=\operatorname{co}\left\{z:|z| \leq \max \left\{\max \left\{\frac{\left|\tilde{c}_{j j}\right|}{h}\right\}, \max \left\{\frac{\left|\tilde{c}_{j-1, j-1}\right|+\left|\tilde{c}_{j+1, j+1}\right|}{2 h}\right\}\right\}\right\} \\
=\left\{z:|z| \leq \max \left\{\frac{\left|\tilde{c}_{j j}\right|}{h}\right\}\right\}
\end{array}
$$

by using the fact that diagonal entries of $\tilde{C} D_{0}$ are zero. Combining this result with field of values of $i \tilde{L}_{h}$, we obtain

$$
\sigma(A) \subset F(A) \subset-i\left[0, \tilde{\lambda}_{M}\right]+\left\{z:|z| \leq \max \left\{\frac{\left|\tilde{c}_{j j}\right|}{h}\right\}\right\}
$$

this is an oval region with axis ratio $O\left(h_{m}^{-1}\right)$ and its major axis lies on imaginary axis.

Remarks The above technique to locate the field of values seems can not sharpened, the results of Hong and Horn [HH91] on Jordan canonical form of products of two hermitean, one of which is semipositive definite, gives insight for the result. Let us write:

$$
-i\left\{\left(D_{\xi}^{-1}\right)^{3} D_{\xi \xi}\right\} D_{0}=\hat{C}\left(i D_{0}\right)
$$

Since $D_{0}$ have purely imaginary eigenvalues (see notes 1 ), then $i \quad D_{0}$ is Hermitean. By the fact that $D_{0}$ also a circulant matrices, then (see Davis book [Da79]):

$$
\text { i } D_{0}=F \Lambda F^{*}
$$

where $\Lambda$ is diagonal matrix with real entries, and $F$ is Fourier matrix, furthermore zero is a simple eigenvalues with multiplicity 1 by inspection [Da79]. Since $\hat{C}$ is diagonal, writes:

$$
\hat{C}=\hat{C}_{+}+\hat{C}_{-}
$$

where $C_{+}$is non-negative diagonal parts of $C$ and $C_{-}$is its corresponding non-positive diagonal parts. Then the field of values of $-i\left\{\left(D_{\xi}^{-1}\right)^{3} D_{\xi \xi}\right\} D_{0}$ can be stated in the Minkowski sum:

$$
F\left(-i\left\{\left(D_{\xi}^{-1}\right)^{3} D_{\xi \xi}\right\} D_{0}\right)=F\left(\hat{C} F \Lambda F^{*}\right) \subset F\left(\hat{C}_{+} F \Lambda F^{*}\right)-F\left(-\hat{C}_{-} F \Lambda F^{*}\right)
$$

So the right hand side is sum of two field of values of product two hermitean matrices, where one of which is symmetric positive definite.

The key point in Hong and Horn [HH91] results that needed for the previous paragraph is that the Jordan form of the product consist of a diagonal blocks of real entries and $r$ tuple of $2 \times 2$ nilpotent block, where $r$ bounded from above by the counts of positive or negative real parts of eigenvalues of each \{factor $\}$. Also it is found that this string of block can not determined a-priorily, this is shown in [HH91] via an 
example that the product may reach the maximum bounds, i.e its Jordan form solely consist of $\left(\frac{n}{2}\right)$ tuple of $2 \times 2$ nilpotent blocks. For our problems at hands, this signify a good and bad luck at the same stroke. The real diagonal blocks signify that the possibility of the field of values will be lies on the real axis $\{$ good result $\}$. But at the same times, the existence of nilpotent blocks, even a single one, will have disastrous effect, since this gives us the field of values of product will be oval region with its major axis lies on the real axis.

Nevertheles, with the grim remarks in the previous paragraphs, we close this remarks by the following guess which some numerical test over several runs confirmed, The eigenvalues region inclusion of the discrete spatial operators is a vertically symmetric box, where the uppermost edge lies on the real axis.

\section{Numerical Check}

For numerical test, we choose the following parameterized transformation:

$$
x(\xi)=\pi \xi+(l-\pi) \operatorname{Sin}(\xi)
$$

from uniform grid $\xi$ over interval $[-\pi, \pi]$ to the non-uniform grid $x$, where $l$ is a parameter. We take $s=64$ grid point for our numerical calculation. To see the parameterization effect to the eigenvalues of discrete spatial operator, we examine only for $l=5.0 e-k, k=1, \cdots, 8$, eventhough extensively we exploring more cases in our numerical run. The main emphasis is to highlights that the eigenvalues of discrete spatial operator never crossing the real axis to take values on the positive imaginary half-plane. In the following Figures we shows subsequently the plots, where in the left hand sides we shows also its field of values region. While on the right hand side, it shows $30 \%$ of the smallest range of its eigenvalues. 

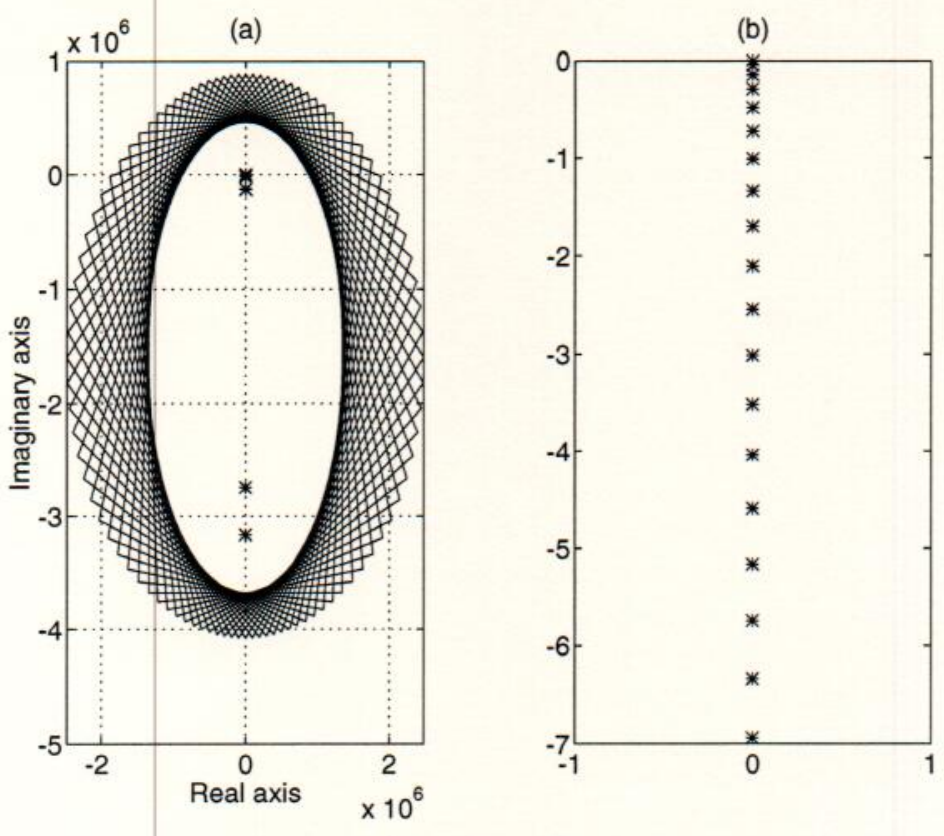

$l=5.0 e-08$

(d)

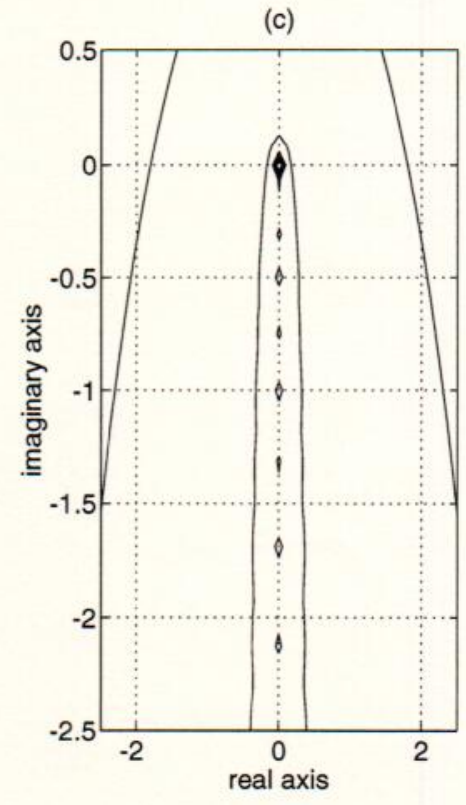




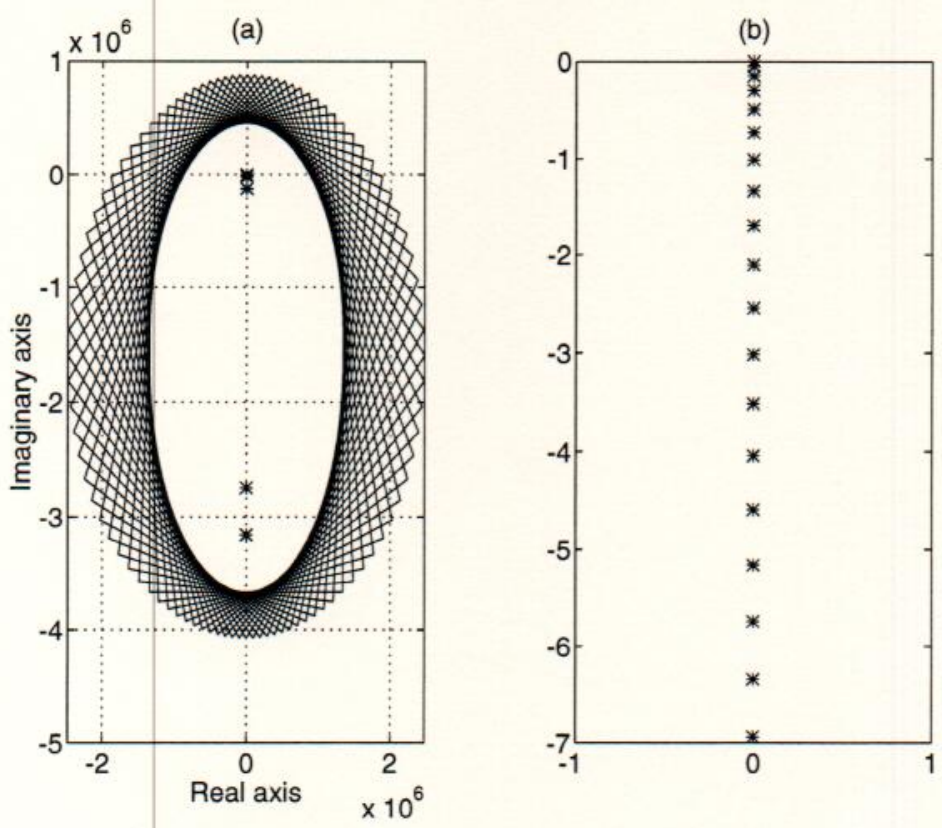

$l=5.0 e-06$

(d)
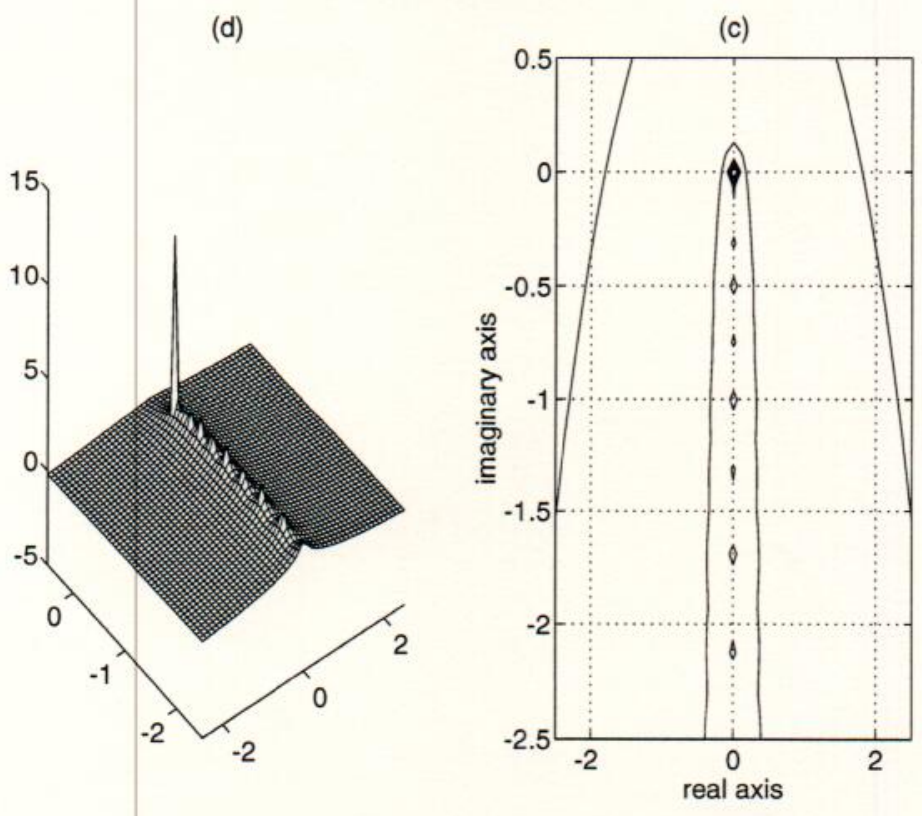

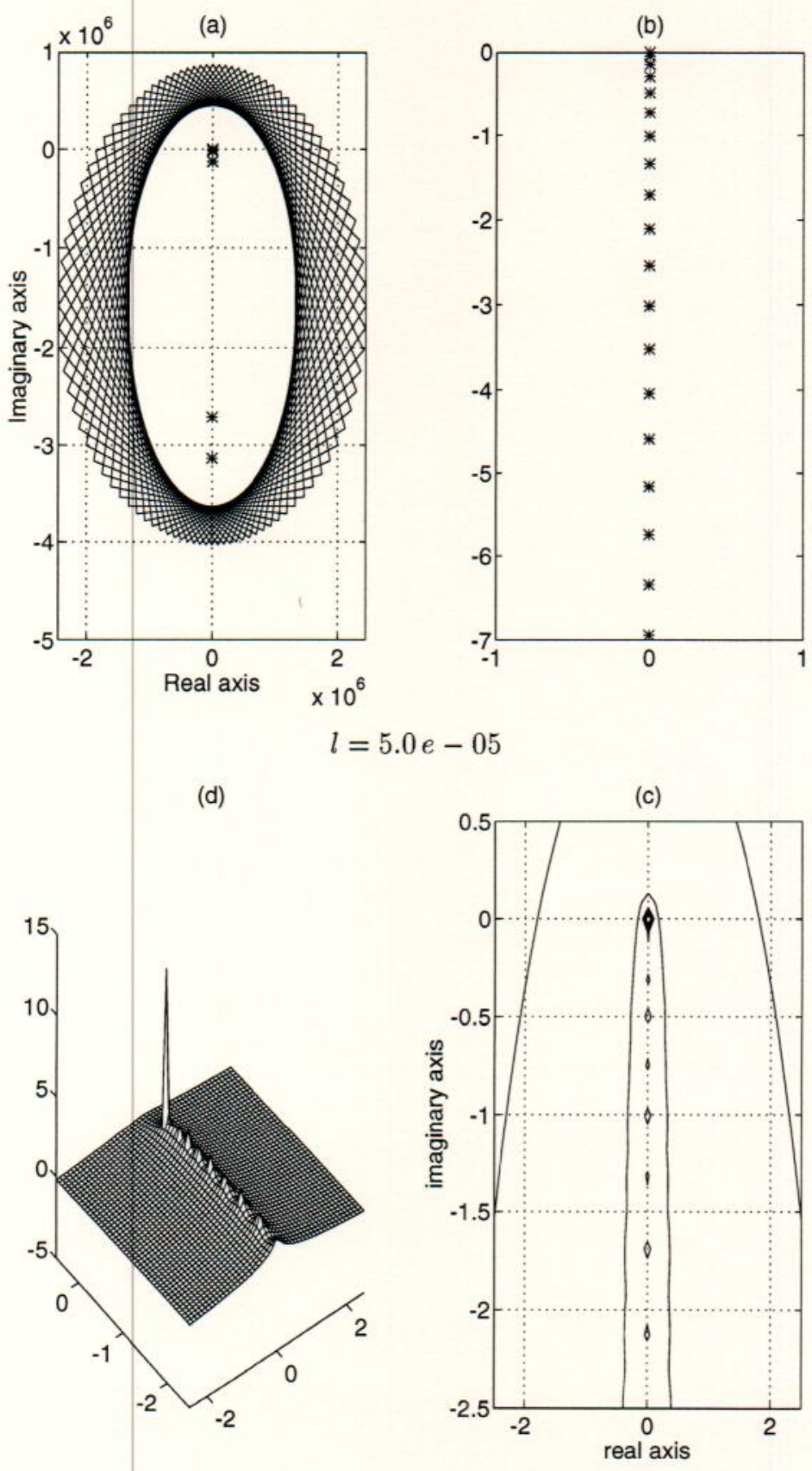


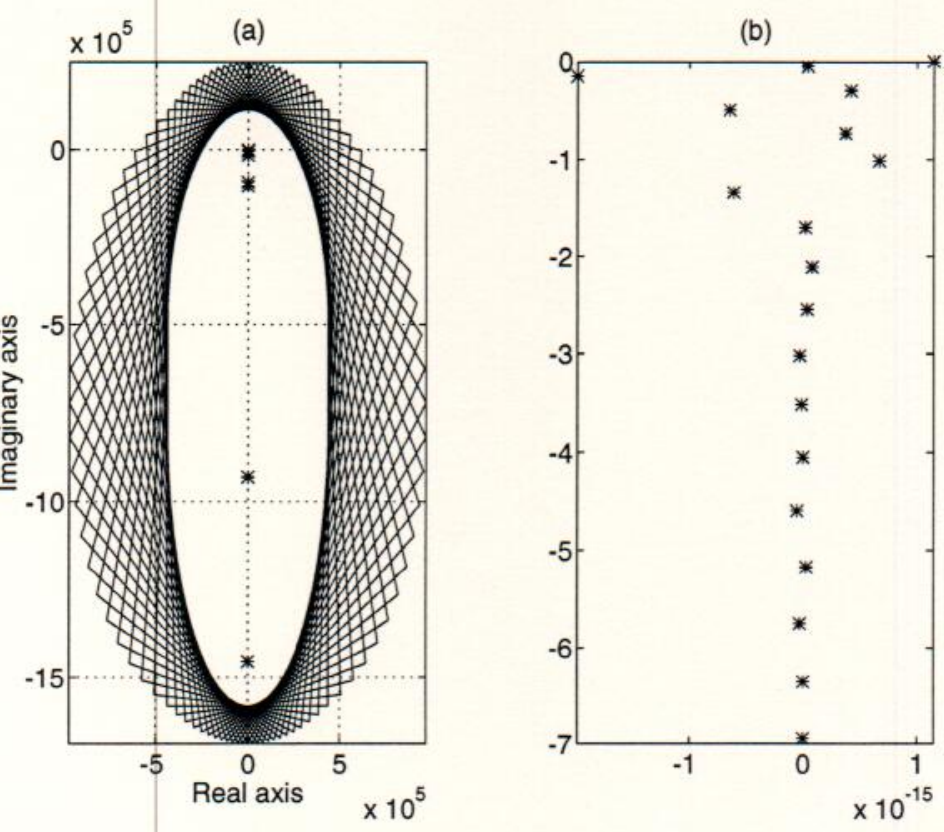

$l=5.0 e-03$

(d)

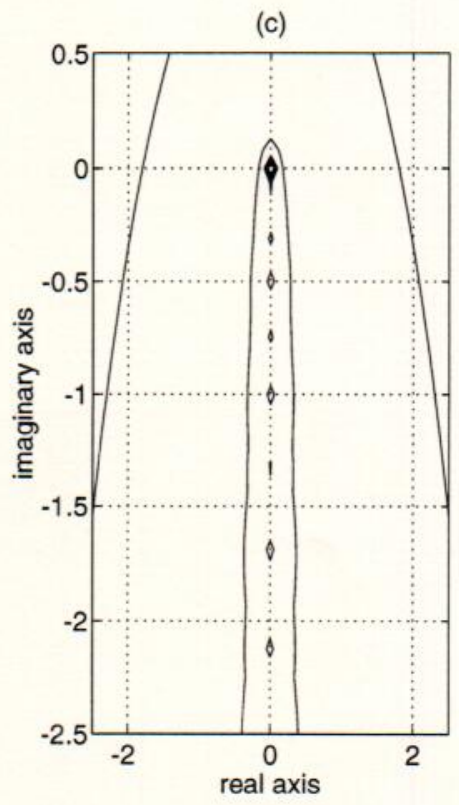



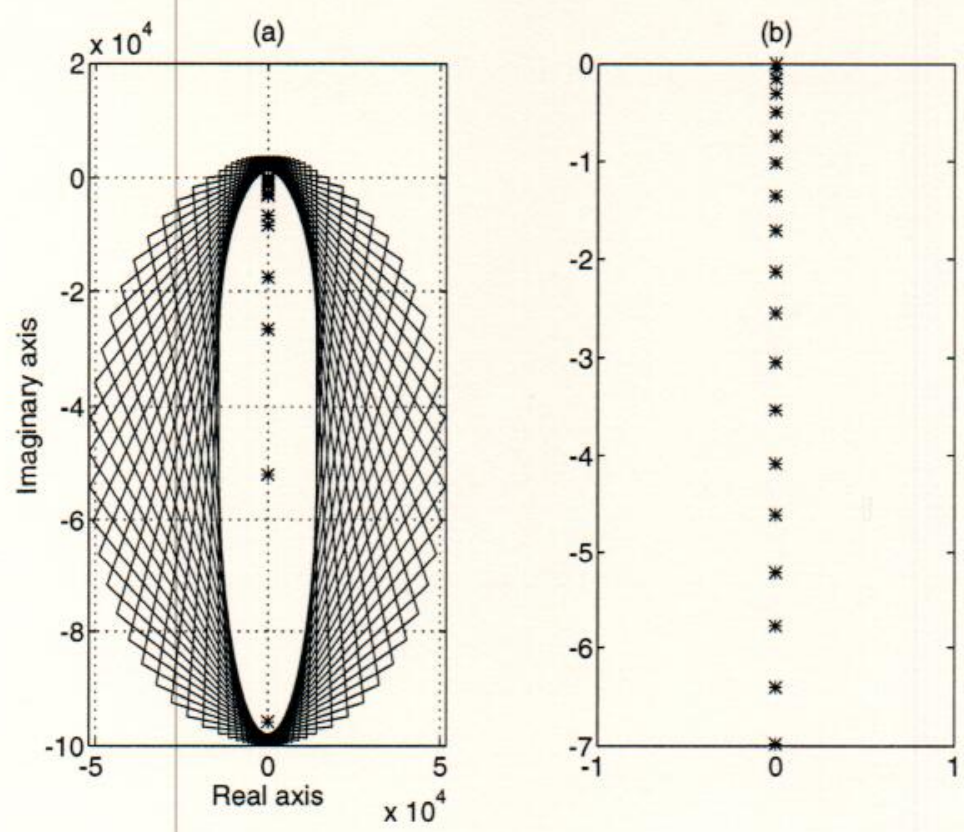

$l=5.0 e-02$

(d)

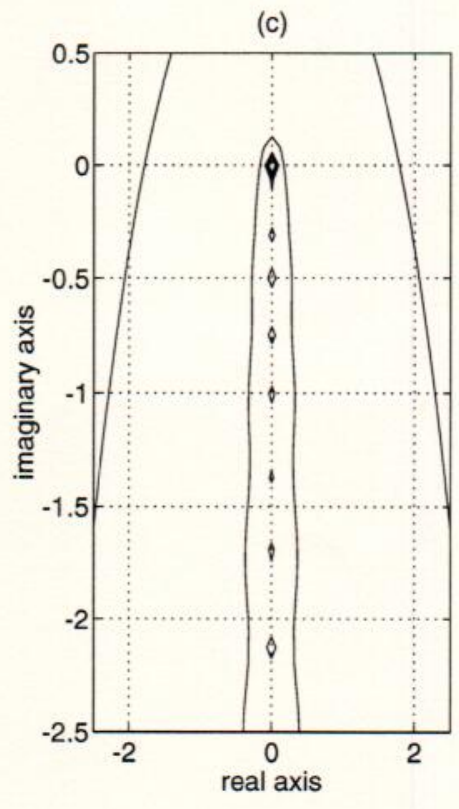


(a)

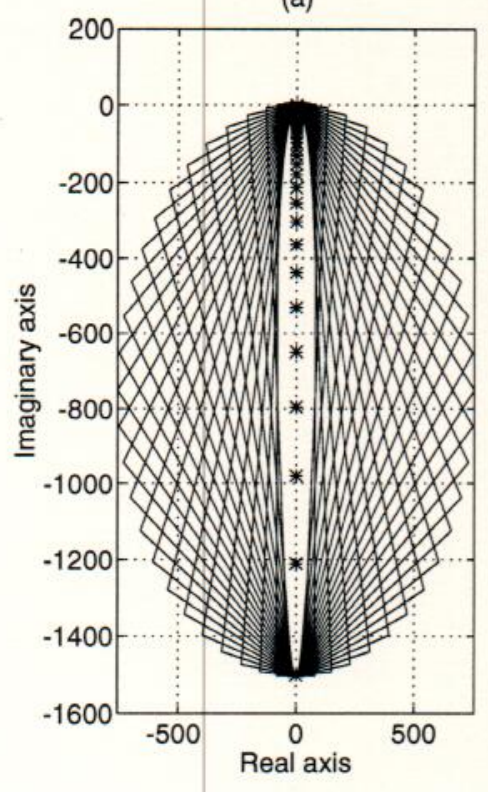

$$
l=5.0 e-01
$$

(d)

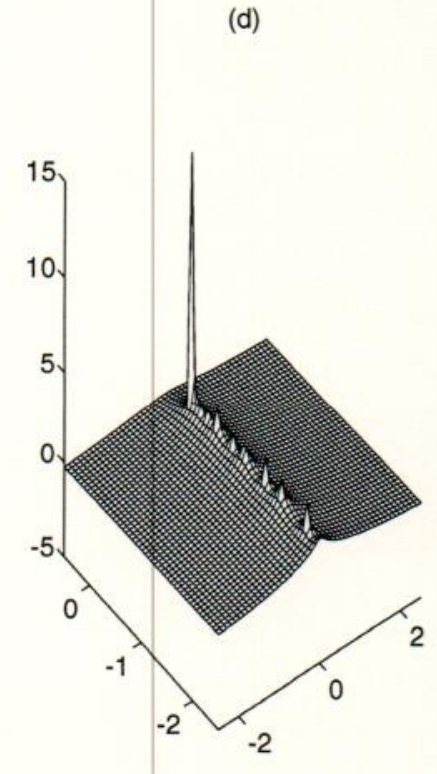

(b)

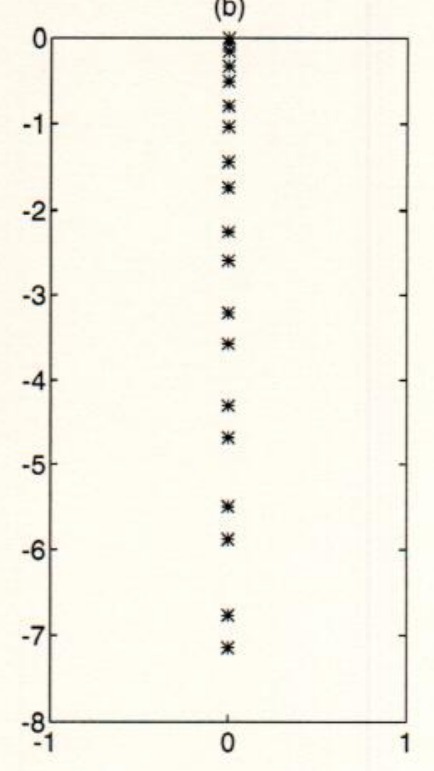

(c)

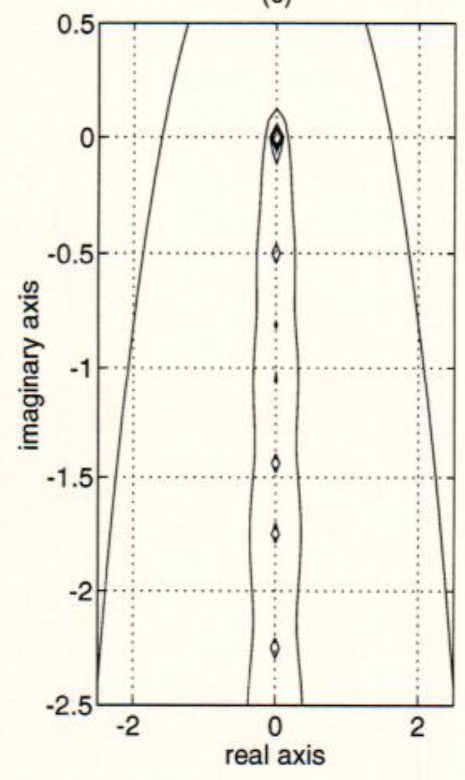




\section{II}

The objective of this note is to investigate stability of backward Euler methods for dynamic grid [Le92] to solve periodic 1-D non-linear Schrödinger :

$$
u_{t}=i u_{x x}+i|u|^{4} u
$$

where $u$ is periodic with periods $2 \pi$, given $2 \pi$-periodic initial values:

$$
u(x, 0)=\phi(x), \phi(x)=\phi(x+2 \pi)
$$

In our analysis, we avoid stability analysis ala vonNeumann using eigenvalue, instead we use logarithmic norm information ( $\mathrm{cf}[\mathrm{Hu} 85][\mathrm{HNW} 87]$ ) to analyze the stability issues. The reason we take this approach is due to non-normality of matrix representation from spatial discretization. This later issue were discussed recently by Higham and Trefethen [HT93] to the related problem for stiff differential equations.

\section{Discretization on Non-Uniform Grid}

Let $\left\{x_{j}\right\}$ denotes a non-uniform grid obtained from a uniform computational grid $\left\{\xi_{j}\right\}$ with spacing grid $h$, using preserving index transformation. Corresponds to nonuniform grid $\left\{x_{j}\right\}$ denotes $h_{m}=\min \left\{h_{j} ; h_{j}=\left(x_{j+1}-x_{j}\right)\right\}$ and $h_{M}=\max \left\{h_{j} ; h_{j}=\right.$ $\left.\left(x_{j+1}-x_{j}\right)\right\}$. Approximating the second and first partial derivatives using center differencing [Fl88], i.e :

$$
\begin{gathered}
\frac{\partial^{2} u}{\partial x^{2}}=\left\{\frac{2 h}{x_{j+1}-x_{j-1}}\right\}^{2} \times \\
\left\{\frac{u_{j+1}-2 u_{j}+u_{j-1}}{2 h^{2}}-\frac{h_{j}-h_{j-1}}{h^{2}} \frac{u_{j+1}-u_{j-1}}{h_{j}+h_{j-1}}\right\} \\
=\left(D_{\xi}^{-1}\right)^{2} \times\left\{L_{h}-D_{\xi \xi} D_{\xi}^{-1} D_{0}\right\} u
\end{gathered}
$$

where $D_{\xi \xi}$ and $D_{\xi}$ are diagonal matrices with entries : $\left(\frac{x_{j+1}-2 x_{j}+x_{j-1}}{h^{2}}\right)=\frac{h_{j}-h_{j-1}}{h^{2}}$ and $\left(\frac{x_{j+1}-x_{j-1}}{2 h}\right)=\frac{h_{j}+h_{j-1}}{2 h}$ respectively. The discrete problem, now can be written in the matrix form :

$$
\frac{d u}{d t}=i\left(D_{\xi}^{-1}\right)^{2}\left\{L_{h}-D_{\xi \xi} D_{\xi}^{-1} D_{0}\right\} u+f(u)
$$

where $f(u)=i B[u] u, B[u]=\operatorname{diag}\left(\left|u_{1}\right|^{4}, \ldots,\left|u_{s}\right|^{4}\right)$. We may rewrite it after regrouping :

$$
\frac{d u}{d t}=i\left(D_{\xi}^{-1}\right)^{2} L_{h} u-i\left\{\left(D_{\xi}^{-1}\right)^{3} D_{\xi \xi}\right\} D_{0} u+f(u)
$$

So the dynamic grid can be represented in view of method of lines as:

$$
\frac{d u}{d t}=i A(t) u(t)+f(u)
$$

Using step-wise solver, we have to solve the following recurrence problem:

$$
u(n+1)=\phi(\tau A(n)) u(n)+p(n), n=0,1, \cdots
$$


where $\tau$ is time step and $\phi$ is rational function that represents the method.

We concerned with stability of the following linear recurrence :

$$
u(n+1)=\phi(\tau A(n)) u(n), n=0,1, \cdots
$$

with property of eigenvalues of $\lambda(A(n))$ :

$$
\begin{gathered}
0<|\Re(\lambda(A(n)))|<O\left(h_{m}(n)\right)<<1 \\
0>\Im(\lambda(A(n)))>-\left|\lambda_{M}(n)\right|=O\left(h_{M}^{-1}\right)
\end{gathered}
$$

It is clear from here that its logarithmic norm induced by inner-product in $\mathbf{C}^{s}$ is: $\mu[A]=\max \Re(\lambda(A(n)))<O\left(h_{m}(n)\right)<<1$ is non-negative.

\section{Stability Estimates}

First, let us consider for 'frozen' coefficient problem, i.e a recurrence problem with fixed A. Let the matrix norm be induced by inner products in $\mathbf{C}^{s}$ We can show that the linear recurrence problem with fixed $\mathrm{A}$ is weakly-stable. This is due to the following result given in Brenner \& Thomée [BT79].

Theorem

Suppose that:

$$
\left|e^{t A}\right| \leq \gamma_{0} e^{\mu t}, t \geq 0
$$

for some $\mu>0, \gamma_{0}>0$.

For each rational function $r$ with $|r(z)| \leq 1$ for $\Re(z) \leq 0$, there are constants $\gamma_{1} \& \kappa$ such that:

$$
\left|r^{n}(\tau A)\right| \leq \gamma_{0} \gamma_{1} \sqrt{n} e^{\mu \kappa T}, T=n \tau
$$

For proof see [BT79]

For backward Euler, the rational function of recurrence relations are given by:

$$
\phi(t)=\frac{1}{1-t}
$$

Since $\mu[A]$ is non-negative, it is clear that :

$$
\left|e^{t A}\right| \leq \gamma_{0} e^{\mu[A] t}, t \geq 0
$$

with $\gamma_{0}=1$. So the linear recurrence problem with frozen A satisfy the required assertion, hence:

$$
|\phi(\tau A)| \leq \gamma_{1} \sqrt{n} e^{\mu[A] \kappa T}, T=n \tau
$$

Denote by $\mu[A(n)]$ is logarithmic norms of $A(n)$.

Lemma Let $\tau$ be given with $0<\tau<\infty$ and $\tau \mu[A(n)]<1$. Then $\phi(\tau A(n))$ exists

\section{Proof}

By Hundsdorfer [Hu85] p15, Lemma 2.2.6

Theorem Let $\tau$ and T be given with $0<\tau<\infty, 0<\tau<\infty$ and $T=N \tau$. Let $m(n)=\mu[A(n)]$. Then backward Euler for method of lines representation of discretized NLS is stable. 
Proof

$$
\begin{gathered}
u_{n+1}=\phi(\tau A(n)) u_{n} \\
\left|u_{n+1}\right| \leq|\phi(\tau A(n))|\left|u_{n}\right|
\end{gathered}
$$

\{ by Hundsdorfer, pp ??\}

$$
\begin{gathered}
\left|u_{n+1}\right| \leq|\phi(\tau m(n))|\left|u_{n}\right| \\
\leq \frac{1}{(1-\tau m(n))}\left|u_{n}\right|
\end{gathered}
$$

\{ by Hundsdorfer, pp ??\}

doing recursively we have:

$$
\left|u_{n+1}\right| \leq(1+\tau m(n))\left|u_{n}\right|, \text { for } \tau m(n) \leq \frac{1}{2}
$$

$$
\begin{aligned}
& \left.\left|u_{n+1}\right| \leq \Pi_{k=1}^{n}(1+\tau m(k))\right)\left|u_{0}\right| \\
& \leq \exp \left(\sum_{k=1}^{n} \ln (1+\tau m(k))\right)\left|u_{0}\right| \\
& \leq \exp \left(\sum_{k=1}^{n} \tau m(k)\right)\left|u_{0}\right|
\end{aligned}
$$

since $\ln (1+\tau m(k)) \leq \tau m(k)$.

where $\hat{m}=\sum_{k=1}^{n} \max \{0, m(k)\}, T=N \tau$.

$$
\left|u_{n+1}\right| \leq \exp (\hat{m} T)\left|u_{0}\right|, \text { for every } n=0,1, \ldots, N-1
$$

Theorem Let $\mu[A(t)]=m(t)$ and $m_{+}(t)=\max \{0, m(t)\}$. Assume that $m_{+}(t) \leq$ $K(t)$, where $\Gamma=\int_{t_{0}}^{\infty} K(t)<\infty$ hold for monotonically decreasing function $\mathrm{K}(\mathrm{t})$. Then backward Euler for method of lines representation of discretized NLS is uniformly stable.

Proof Sketch of proof

$$
\begin{gathered}
u_{n+1}=\phi(\tau A(n)) u_{n} \\
\left|u_{n+1}\right| \leq|\phi(\tau A(n))|\left|u_{n}\right|
\end{gathered}
$$

\{ by Hundsdorfer, pp ??\}

$$
\begin{aligned}
& \leq|\phi(\tau m(n))|\left|u_{n}\right| \\
& \leq \frac{1}{(1-\tau m(n))}\left|u_{n}\right|
\end{aligned}
$$




$$
\begin{gathered}
\leq 1+\frac{\tau m_{+}(n)}{(1-\tau m(n))}\left|u_{n}\right| ; \tau m(n) \leq 1-\gamma, \text { forsome } \gamma>0 \\
\leq \exp \left(\frac{1}{\gamma} \sum \tau m(n)\right)\left|u_{0}\right| \\
\leq C\left|u_{0}\right|
\end{gathered}
$$

, where: $C=\exp \left(\frac{\Gamma}{\gamma}\right)$ 


\section{Bibliography}

[Ax92] O. Axelsson, 1992, Bounds of Eigenvalues of Preconditioned Matrices , SIAM J. Matrix Anal. Applic., v 13, 847-862

[BT79] P. Brenner \& V. Thomée, 1979, On Rational Approximations of Semigroups, SIAM J. Num. Anal., v 16, 683-694

[Da79] P. J. Davis, 1979, Circulant Matrices, John-Wiley \& Sons

[F188] C. A. J. Fletcher, 1988, Computational Techniques for Fluid Dynamics, Vol II, Springer-Verlag

[Fr93] R. Freund, 1993, A Tranpose-Free Quasi-Minimal Residual Algorithm for non-Hermitean Linear Systems, SIAM J. Sci. Comp., v 14, 470-482

[HJ91] R. Horn \& C. R. Johnson, 1991, Topics in Matrix Analysis, Cambridge University Press

[HH91] Y. Hong \& R. A. Horn, 1991, The Jordan Canonical Form of A Product of a Hermitean and a Positive semi-definite matrix, Lin. Alg. Applic., v 147, 373-386

[HNW87] E. Hairer S. P. Norsett \& G. Wanner, 1987, Solving Ordinarry Differential Equations I. Nonstiff Problems, Springer-Verlag

[HT93] D. J. Higham \& L. N. Trefethen, 1993, Stiffnes of ODEs, BIT, v 33, 285-303

[Hu85] W. H. Hundsdorfer, 1985, The Numerical Solution of Nonlinear Stiff Initial Value Problems: An Analysis of One Step Methods, Doctorate Dissertation, University of Leiden. The Netherlands. \{ Reissued as CWI-Tracts Number 12 \}

[Le92] B. LeMesurier, 1992, A New Dynamic Adaptive Grid Numerical Approach for Non-Isotropic Focusing Solutions of the Nonlinear Schrödinger and other nonLinear Evolution Equations, Technical Reports CMA-MR10-92, CMA-ANU

[RT92] S. C.Reddy \& L. N. Trefethen, 1992, Stability of The Method of Lines, Numer. Math, v 62, 235-267

[RT92] L.Reichel \& L. N. Trefethen, 1992, The Eigenvalues and Pseudo-Eigenvalues of Toeplitz Matrices, Lin. Alg. Applic., v 162, 153-185

[SS92] J. Sands \& S. Skelboe, 1992, Stability of Backward Euler Multirate Methods and Convergence of Waveform Relaxation BIT, v 32, 350-366 